\section{Systemischer Lupus erythematodes: Netzhauttoxizität von Antimalariamittel}

\footnotetext{
Mukwikwi ER et al. Retinal Complications in Patients With Systemic Lupus Erythematosus Treated With Antimalarial Drugs. J Rheumatol 2020; 47: 553-556
}

Hydroxychloroquin (HCQ) und Chloroquin (CQ) sind Schlüsselmedikamente zur Behandlung des systemischem Lupus (SLE) und ähnlichen Erkrankungen. Die Netzhauttoxizität ist die besorgniserregendste Nebenwirkung. Kanadische Ärzte untersuchten Faktoren, die möglicherweise mit der Toxizität der Netzhaut zusammenhängen, mithilfe von Fall-Kontroll-Analysen.

Die Grundlage ihrer Studie bildeten Daten von Patientinnen und Patienten mit der Diagnose SLE, die in der Datenbank des Gesundheitszentrums der McGill Universität in Montreal registriert waren. Aus dieser SLE-Klinikkohorte identifizierten sie Patientinnen und Patienten mit Netzhautveränderungen anhand des Systemic Lupus International Collaborating Clinics/American College of Rheumatology (SLICC/ACR) Da- mage Index (SDI). Die Hydroxychloroquin und Chloroquin bedingten Retinopathien wurden durch einen Augenarzt anhand verschiedener Untersuchungen und Tests bestätigt. Jedem Fall wurden 3 SLE-Kontrollen gegenübergestellt, die mit HCQ/CQ behandelt worden waren und keine Retinopathien entwickelten und die nach Alter bei SLEDiagnose und SLE-Dauer übereinstimmten.

Von 362 Patientinnen und Patienten, die bis zum Jahr 2016 erfasst wurden, wurden 326 (90\%) für mindestens 1 Jahr mit Antimalaria behandelt. Während einer durchschnittlichen Nachbeobachtungszeit von 12,8 Jahren entwickelten 18 (5,5\%) dieser $326 \mathrm{~Pa}$ tientinnen und Patienten eine Netzhauttoxizität. Insgesamt wurde die Mehrheit (90\%) nur mit Hydroxychloroquin behandelt, 31 Personen wurden mit HCQ und CQ behandelt.

Die Mindestanzahl von Jahren HCQ/CQ-Exposition bis zur Entwicklung einer Retinopathie betrug 8 Jahre und die maximale Expositionsdauer 33 Jahre. Die mediane HCQ/ CQ-Expositionsdauer war in den Retinopathiefällen und den Kontrollen vergleichbar (19 Jahre, Interquartilbereich [IQR] 14-20] vs. 16 Jahre, IQR 11-22). Dies beruhte wahrscheinlich auf dem Matching der Vergleichsgruppen unter Einbeziehung der SLE-Dauer.

Im Vergleich zu den Kontrollpersonen, fanden sich bei den Patientinnen und Patienten mit Netzhauttoxizität tendenziell mehr Nierenerkrankungen (22,2 vs. 14,8\%) und in der Gruppe Netzhauttoxizität waren auch etwas weniger Personen weiß $(61,1$ vs. 74,1\%). Allerdings erreichte keine dieser Variablen statistische Signifikanz.

Alle Patientinnen und Patienten mit Retinopathie sowie 44 Kontrollpersonen (81,5\%), die nach mindestens einem Jahr überprüft wurden, waren mit höheren HCQ/CQ-Dosen, als in den Leitlinien empfohlen, behandelt worden.

Bei den Patientinnen und Patienten mit Retinopathie war der Anteil derjenigen, die zuvor mit CQ behandelt worden waren, mehr als dreimal so hoch wie der bei den Kontrollpersonen.
FAZIT

Etwas mehr als 5\% der SLE-Patientinnen und Patienten entwickelten über einen Zeitraum von durchschnittlich 12,8 Jahren Netzhautkomplikationen durch die Verwendung von Antimalariamittel. In den ersten 5 Jahren der Therapie wurden keine Retinopathiefälle festgestellt. Die Verwendung von CQ führte zu deutlich mehr Komplikationen. Derzeit finden Studien mit größeren Populationen statt, um die Rolle von Therapiedauer, der Ethnie sowie weiterer Faktoren besser zu deuten, schreibt das Autorenteam.

Richard Kessing, Zeiskam 